\title{
Nanoscale
}

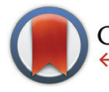

CrossMark

$\leftarrow$ click for updates

Cite this: Nanoscale, 2015, 7, 7155

Received 9th January 2015,

Accepted 17th March 2015

DOI: $10.1039 / c 5 n r 00154 d$

www.rsc.org/nanoscale

\section{Magnetic field assisted assembly of highly ordered percolated nanostructures and their application for transparent conductive thin films $\uparrow$}

\author{
Oleksandr Trotsenko, Alexander Tokarev, Alexey Gruzd, Timothy Enright and \\ Sergiy Minko*
}

directed assembly of nanoparticulates ${ }^{12}$ uses field-induced polarization and strong dipole-dipole interactions to guide particle assembly into desired configurations. ${ }^{13}$ The method of magnetophoretic assembly has been successfully applied for building nanostructured materials with enhanced properties: superhydrophobic structures, ${ }^{14}$ aligned carbon nanotubes composites,${ }^{15-18}$ and magnetically controllable photonic crystals. ${ }^{19}$ It was shown that anisotropic coatings could be deposited and realigned using the remotely controlled magnetic forces experienced by nanoparticulates. ${ }^{20-23}$ Kinetics of magnetophoresis depends on the size of particulates. Magnetic forces acting on nanoparticles with a diameter below $10 \mathrm{~nm}$ in the inhomogeneous magnetic field in vicinity of a $1 \mathrm{~T}$ magnet, are weak to compete with Brownian motion. However, alignment and assembly of particles in chain-like structures results in substantial amplification of the response of magnetic assemblies to inhomogeneous magnetic fields. ${ }^{24,25}$ This simple approach of assembling magnetic particles into linear aggregates, or deposition of magnetic nanoparticles on the surface of non-magnetic nanostructures, becomes a powerful tool to control transport and alignment of nanostructures in magnetic fields generated by ordinary permanent magnets.

The successful attempts mentioned above call for further development of new approaches for field-assisted assembly of nanostructured building blocks into functional materials and devices. An example of such nanostructured materials are flexible, transparent conductors assembled using metal nanowires that have numerous applications. Transparent, solid and flexible conductors with a sheet resistance ranging from $10 \Omega \mathrm{sq}^{-1}$ to $10^{6} \Omega \mathrm{sq}^{-1}$ and transparency $\geq 90 \%$ find various applications in electronic devices for antistatic coatings (at $10^{6} \Omega \mathrm{sq}^{-1}$ ), touch screens (at 400-1000 $\Omega \mathrm{sq}^{-1}$ ), OLEDs and solar cells electrodes (at $\leq 10 \Omega \mathrm{sq}^{-1}$ ). ${ }^{26}$ Transparent conductors made of silver nanowire networks possess unique and superior properties such as mechanical flexibility, optoelectronic performance, and chemical stability. ${ }^{26-31}$ Traditionally, conductive coatings are fabricated by deposition of silver nanowires via Mayer rod coating method, ${ }^{28}$ spray coating, ${ }^{32,33}$ vacuum filtration coating on membranes, ${ }^{34}$ and electrospinning. ${ }^{27}$
Nanostructured Materials Laboratory, the University of Georgia, Athens, GA 30602, USA.E-mail: sminko@uga.edu

$\dagger$ Electronic supplementary information (ESI) available. See DOI: 10.1039/ c5nr00154d 
Although high-quality coatings are provided, traditional methods result in random deposition of the conductive high aspect ratios particulates.

Randomly generated, percolating conducting structures are not optimal in terms of combining the highest transparency and the highest conductivity. Some fraction of randomly deposited conductors are not a part of the electric circuit because of defects in electrical contacts, i.e. dangling ends and loops that are found with nanoelectrical atomic force microscopy (AFM) imaging (see Fig.S1, ESI $\dagger$ ). Such defects in the material do not contribute to conductivity but negatively affects transparency. Alignment of conductive nanowires to generate an anisotropic percolated network with fewer defects could substantially improve electrical and optical properties of the coatings. Hong et al. ${ }^{35}$ fabricated square grids of silver nanowires by laser sintering metal nanoparticle ink. They demonstrated that networks with well-defined structure possess superior properties. However, the method is limited to silver lines a few micrometers in width and about $100 \mathrm{~nm}$ in height. Improvements to optical and electrical properties of transparent electrodes could be approached by reducing dimensions of the conductive regular grids. $^{36}$ Various approaches were explored for alignment of gold and silver nanowires in thin films and bulk materials including Langmuir-Blodgett method, ${ }^{37-40}$ deposition on micropatterned silicon, ${ }^{41}$ mechanical deformation of polymer matrix ${ }^{42}$ electric field, ${ }^{43}$ linear $\lambda$-DNA templates, ${ }^{44}$ chemical amidation reaction on $\operatorname{Si}(100)$ surface $^{45}$ and electrochemical deposition methods. ${ }^{46,47}$

\section{Results and discussion}

The goal of this work is to develop scalable magnetic field directed assembly of conductive nanowires for generation of aligned conductive networks that could be applied on relatively large (macroscopic) surface areas. In our approach, non-magnetic silver nanowires are decorated with sacrificial magnetic iron oxide nanoparticles. Polyethyleneimine (PEI) is used to "glue" iron oxide superparamagnetic nanoparticles to silver nanowires through electrostatic and van der Waals interactions (Fig. 1a, d and g). A magnetic field gradient is used to deposit nanowires on the surface of a transparent substrate (glass or plastic) (Fig. 1b, c, e and f). It is noteworthy that solvent and substrates have no substantial effect if the particle dispersion is stable during deposition. The experiments demonstrated an optimal weight ratio of nanowires to magnetic nanoparticles ranging from $1: 1$ and $2: 1$. In that range of particle ratios, silver nanowires are decorated by adsorbed superparamagnetic particles and form composite magnetic nanowires. A deviation from this ratio results in slow deposition kinetics and aggregation of the particle dispersions.

In the experiments, a glass slide or a transparent plastic film is placed in a container filled with a diluted dispersion of silver nanowires decorated with magnetic particles in ethanol or water. A permanent magnet is placed under the substrate outside the container (see setup in Fig. S2, ESI $\dagger$ ). The position of the magnet is adjusted to optimize an appropriate combination of the strong tangential $\left(B_{x}\right)$ and weak normal $\left(B_{z}\right)$ components of the magnetic field vector. During deposition, the tangential component of the magnetic field is constant in the plane parallel to the sample within the area of the sample. $B_{x}$ created magnetic torque on the nanowires that aligns them along the $X$-direction (in the plane of the transparent substrate). The gradient of the magnetic field in vertical $Z$-direction is $10.7 \mathrm{~T} \mathrm{~m}^{-1}$. The gradient of the magnetic field generates the force which drives composite magnetic nanowires to the surface of the glass slide (Fig. 1b, e, c, and f; Fig. S3, ESI $\dagger$ ). If the tangential component was weak, unaligned mesh structures were deposited (Fig. S4, ESI†)). Following the complete deposition of silver nanowires and removal of the supernatant, a small amount of surfactant was added to reduce capillary force effects, and the sample was dried in the magnetic field.

A regular grid of nanowires was fabricated in two consecutive steps. After deposition of the first layer of silver nanowires, the sample was dried and placed in a container with a fresh dispersion of nanowires. The horizontal component of the magnetic field vector was rotated $90^{\circ}$ and the second layer of nanowires was adsorbed on the surface (Fig. 1c and f). Once the coating was dried, it became stable to changes in field directions even when immersed in a liquid. Optical properties of the deposited conductive network were improved by etching the iron oxide particles with oxalic acid solution ${ }^{48}$ (Fig. $1 \mathrm{~h}$ and j). Further improvement of film conductivity was achieved by annealing at $150-200{ }^{\circ} \mathrm{C}$ for $20-30 \mathrm{~min}$ to fuse silver junctions. ${ }^{49,50}$ Fusing enhances flexibility and mechanical stability of networks so that the structures can be used on flexible substrates or transferred onto other substrates ${ }^{32,51-53}$ (see Fig. S5, ESI $\dagger$ ).

Conductive and optical properties of silver nanowire films depend dramatically on the size of the nanowires. The experimental results were collected using the same stock sample of silver nanowires for the characterization of the aligned network versus a random network. Characteristics of the obtained transparent conductors are summarized in Fig. 2.

The results for the aligned structures were compared with control samples of the conductive coatings prepared using a random deposition as described by De et al. ${ }^{34}$ Alignment of silver nanowires in networks (Fig. 2c) was characterized by measuring the orientation of more than 100 different nanowires in terms of angular coordinates using SEM and AFM images with image analysis software (ImageJ). The angular coordinates for unaligned samples were randomly distributed, while aligned networks clearly demonstrated preferential orientation in direction of the tangential magnetic field component. Regular grid samples demonstrated the orientation of wires along the two orthogonal vectors of tangential magnetic field components in each deposition step, respectively. The aligned nanowire meshes demonstrate superior properties in comparison to random structures (Fig. 2b). For example, the sheet resistance of $100 \Omega \mathrm{sq}^{-1}$ resulted in $87 \%$ transparency of 

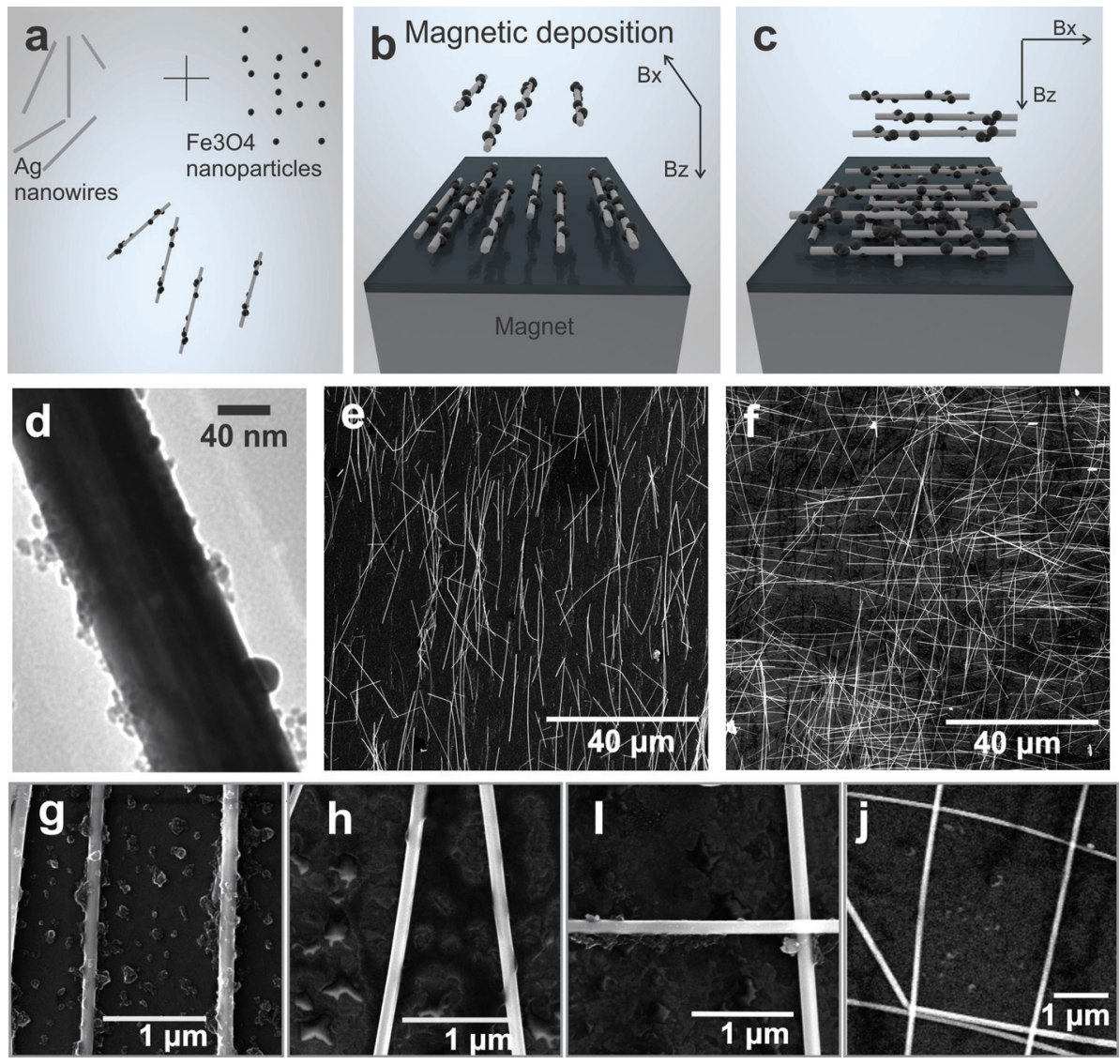

Fig. 1 Fabrication of aligned networks of silver nanowires: (a) dispersions of silver nanowires and iron oxide nanoparticles are mixed to bind magnetite nanoparticles to nanowires; iron oxide particles on the surface of silver nanowires are visualized with TEM (d) and SEM (g). (b) A permanent magnet is placed under the substrate and the inhomogeneous magnetic field directs nanowires toward the substrate when the tangential component of the magnetic field aligns nanowires as visualized with SEM (e, g, h). (c) The second layer is deposited when the magnet is rotated 90 degrees; SEM images ( $f, i, j)$. After deposition the magnetite nanoparticles are etched (SEM images $h$, i). The network is annealed at $150-200{ }^{\circ} \mathrm{C}$ for 20-30 minutes (SEM image J).

the aligned coating, whereas, the random coating leads to $72 \%$ transparency. Furthermore, for an equal transparency of $72 \%$, the aligned network has a sheet resistance of only $10 \Omega \mathrm{sq}^{-1}$.

Transmittance $T(\lambda)$ of the aligned silver nanowire networks measured in the visible spectral range show a linear dependence of $T(\lambda)$ on the nanowire density (Fig. 2d). The best transmittance achieved is greater than $90 \%$. Quality of transparent electrodes can be quantitatively estimated using the figure of merit of transparent electrodes ${ }^{50}$ :

$$
T(\lambda)=\left(1+\frac{188.5}{R_{\mathrm{sh}}} \frac{\sigma_{\mathrm{Op}}(\lambda)}{\sigma_{\mathrm{DC}}}\right)^{-2}
$$

where $\sigma_{\mathrm{DC}}$ is the dc conductivity, and $\sigma_{\mathrm{Op}}$ is the optical conductivity

The ratio $\sigma_{\mathrm{DC}} / \sigma_{\mathrm{Op}}$ is a figure of merit obtained by fitting the experimental data (Fig. 2b). The obtained $T$ values of 93 and 13 for aligned and random networks, respectively, demonstrate substantial advantage of the aligned structures. Sheet resistance (Fig. 2a) and visible range transmittance (Fig. 2b) as a function of nanowire concentration in the coatings can be dis- cussed using the percolation theory of metal-insulator heterogeneous system. ${ }^{54}$ Electric current in a metallic percolation cluster is approached when the metallic phase density on the surface $(N)$ approaches the percolation threshold $\left(N_{\mathrm{c}}\right)$. At $N<$ $N_{\mathrm{c}}$, the infinite cluster breaks down into finite fragments and probability of percolation rapidly drops. An optimal conductivity to transparency ratio could be achieved by minimizing the percolation concentration. The percolation theory predicts the conductivity $(\sigma)$ dependence as $\sigma \propto\left(N-N_{\mathrm{c}}\right)^{t}$, where $t$ is the critical conductivity exponent. For the sheet resistance of the coatings $\left(R_{\mathrm{sh}}\right)$, and the silver nanowire concentration (density) on the surface $\rho$ in units of $\mathrm{mg} \mathrm{m}^{-2}$, the equation transforms to $R_{\mathrm{sh}} \propto\left(\rho-\rho_{\mathrm{c}}\right)^{-t}$. The sheet resistance measurements (Fig. 2a) show that the percolation concentration for aligned networks deposited by magnetophoresis and the control random sample occurs at about $12 \mathrm{mg} \mathrm{m}^{-2}$ and $20 \mathrm{mg} \mathrm{m}^{-2}$, respectively (see ESI $\dagger$ ). Using the estimated percolation densities, the experimental data were fitted as shown in Fig. 2a and in the inset. The obtained critical exponents $t=2$ and 2.7 for randomly deposited and aligned wires exceed the percolation theory exponents 1.33 and 2 for $2 \mathrm{D}$ and 3D structures, respectively. This 

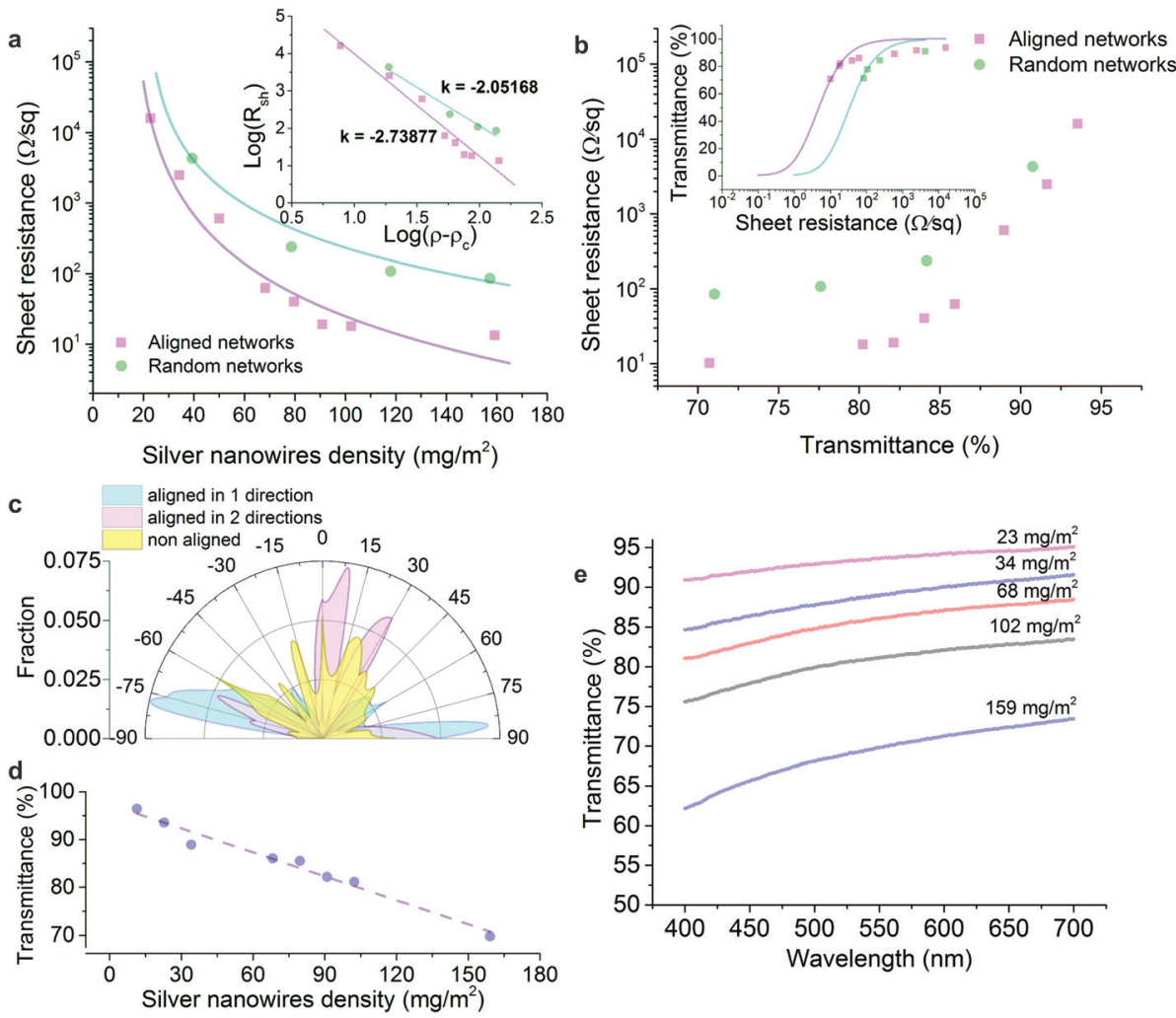

Fig. 2 Transparency and sheet resistance of the aligned vs. random electrodes: (a) sheet resistance vs. silver nanowires density. The solid lines are power function fits. The inset shows the data with linear fits ( $\mathrm{k}$ is the line slope). (b) Sheet resistance vs. transmittance at $550 \mathrm{~nm}$ wavelength. The inset graph shows transmittance vs. sheet resistance and the fitted figure of merit. (c) Distribution of nanowires orientation on the substrate: randomly deposited (yellow), aligned in one (blue) and two (red) directions. Statistics were collected by measuring alignments of 80-120 wires for each sample from SEM and AFM images. (d) Transmittance of aligned silver nanowire networks at $550 \mathrm{~nm}$ as a function of nanowire density. (e) Visible spectra of aligned nanowire networks on glass substrates for different nanowire densities.

deviation could be attributed to the defects in alignment and two types of resistance along the silver nanowires and across wire-wire junctions. ${ }^{28,55-57}$ The defects in alignment are due to the very high aspect ratio of 250 of the silver nanowires which results in bending at the surface deposition stage. Some scattering of angles between orthogonally oriented nanowires could affect the film conductivity. The mesh-like thin films are a few fiber monolayers thick when some fraction of 3D-structures could contribute to the film conductivity. The latter two specific structural aspects are not considered in the 2D percolation network approach. Obviously, the aspect ratio and angles between deposited wires should be optimized to approach the best conductance and optical performance. Doubtless, modelling of electrical conductivity of aligned nanowire meshes at different angles between wires $v s$. randomly deposited 2D and 3D thin films would be very helpful to explain the obtained experimental results.

The scalability of the method is demonstrated in the large area of the coating (Fig. 3). SEM images (Fig. 3b-e) taken in

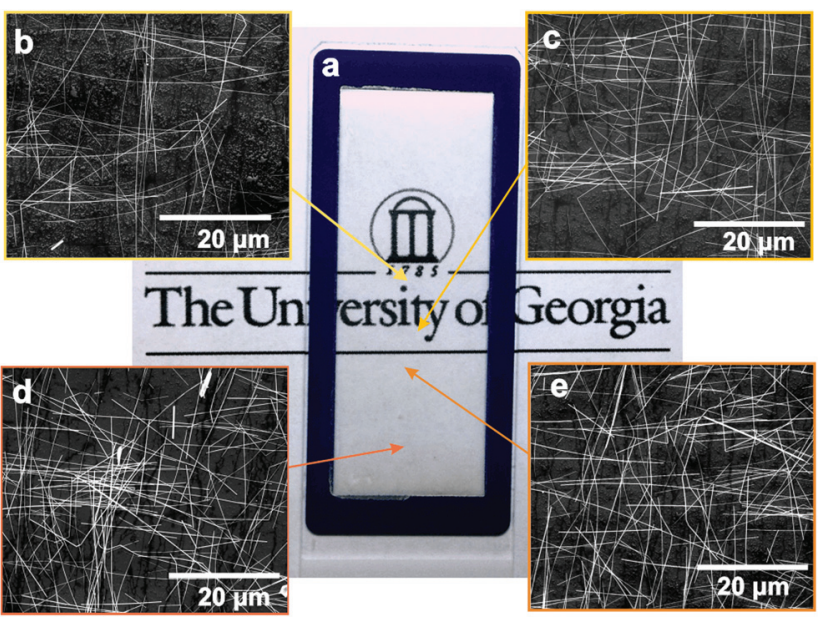

Fig. 3 (a) Optical image of the glass slide with deposited silver wires network after etching of the magnetite nanoparticles. (b-e) Density of wires is quite uniform over large areas as shown on SEM images: $b$ $70 \mathrm{mg} \mathrm{m}^{-2}, \mathrm{c}-90 \mathrm{mg} \mathrm{m}^{-2}, \mathrm{~d}$ and $\mathrm{e}-110 \mathrm{mg} \mathrm{m}^{-2}$. 
different spots show coverage of $90 \pm 20 \mathrm{mg} \mathrm{m}^{-2}$ on the glass slide with dimensions of $1 \times 2$ inches (Fig. $3 \mathrm{~b}$ ). The absolute values of transparency and conductivity depend on the nanowire dimensions, aspect ratio and electrical properties of the conductive material. A screening of commercial samples of nanowires of various dimensions is beyond the scope of this project. In this work, we aimed to demonstrate advantages of the field-directed deposition method for aligned nanostructured coatings $v s$. random deposition.

\section{Conclusions}

In summary, the magnetic field assisted deposition of charge conducting nanowires substantially improves properties of transparent conductive films. Due to the alignment of nanowires in a magnetic field, the highly-ordered networks are achieved with a two-step deposition process. The achieved coatings improved conductivity and transparency characteristics in comparison those obtained by random deposition. The proposed method is simple, scalable, insensitive to solvents, and allows cost-effective fabrication of transparent electrodes.

\section{Experimental section}

\subsection{Materials and methods}

Silver nanowire networks fabrication. Superparamagnetic iron oxide nanoparticles $(13 \pm 4 \mathrm{~nm}$ in diameter, measured with dynamic light scattering, DLS) were synthesized by a coprecipitation method described elsewhere. ${ }^{58}$ The nanoparticles were functionalized by adsorption of PEI and then washed and dispersed in a solvent (water or ethanol) at concentration of $0.1 \mathrm{~g} \mathrm{~L}^{-1}$. Zeta potential of the PEI functionalized nanoparticles was $+40 \mathrm{mV}$ in water at pH6. The silver nanowires with diameter of $90 \pm 20 \mathrm{~nm}$ and average length of $25 \mu \mathrm{m}$, provided by Blue Nano, Charlotte, NC were dispersed in water or ethanol at concentration of $0.1 \mathrm{~g} \mathrm{~L}^{-1}$. Zeta potential of silver nanowires dispersed in water was $-40 \mathrm{mV}$ at pH6. Nanoparticles size and zeta potential of nanoparticles and nanowires were measured by Malvern Zetasizer Nano (see Fig. S6, ESI $\dagger$ ). The dispersions for deposition were prepared by mixing stock $0.1 \mathrm{~g} \mathrm{~L}^{-1}$ dispersions of nanowires and nanoparticles in ratios $1: 1$ or $2: 1$, and subsequently diluting with the solvents to $0.002-0.02 \mathrm{~g} \mathrm{~L}^{-1}$ range of total solid phase concentrations $\left((0.2-2) \times 10^{-6}\right.$ by volume fraction $)$.

A glass substrate was placed into a cuvette and covered with 0.5-4 $\mathrm{mL}$ of dispersion (see Fig. S2, ESI $\dagger$ ). A permanent magnet (NdFeB, Grade N42 by K\&J Magnetics, Pipersville, PA) was placed underneath the sample and outside the cuvette. The exact placement of the magnet is very important. The optimal placement of the magnet was experimentally adjusted in a series of experiments. The wires were typically deposited in a few minutes (see Fig. S7, ESI $\dagger$ for deposition kinetics) and the dispersions turned clear. Density of silver nanowires on the surface of the glass slide was calculated assuming all nanowires from the mixture were deposited onto the substrate. The remaining supernatant was extracted with a syringe, and the sample was dried at ambient conditions. When depositing from water, $25 \mu \mathrm{L}$ of $0.25 \%$ surfactant solution (sodium dodecyl sulfate) was added to the supernatant to lower its surface tension for the drying step. Iron oxide nanoparticle were etched ${ }^{58}$ in a $100 \mathrm{mM}$ oxalic acid and $0.6 \mathrm{mM} \mathrm{FeCl}_{2}$ aqueous solution at pH 2.5 (Fig. $1 \mathrm{~g}$ nanowires before etching and Fig. 1h and Fig. S5c $\dagger$ after etching). The samples were rinsed four times with deionized water and gently dried under argon flow. Finally, the coatings were annealed in an oven at 150-200 ${ }^{\circ} \mathrm{C}$ for $20-30$ minutes.

The control samples were prepared by the deposition of silver nanowires from $0.01-0.02 \mathrm{mg} \mathrm{mL}^{-1}$ dispersions in ethanol on AAO membrane with pore diameter $200 \mathrm{~nm}$ using a syringe filtration setup. After drying the silver nanowire networks were transferred to a glass substrate coated with polydimethylsiloxane (PDMS). The coatings were annealed at the same conditions as the magnetically deposited samples.

\subsection{Optical and electrical characterization.}

Sheet resistance measurements were performed using a home built four point probe assembly using a Keithley power supply, digital milliohmmeter, and C4S-54/5S four-point probe at 3-4 different locations on the sample. Transmittance measurements were carried with the Shimadzu UV-2401 UV-vis spectrometer using a clean glass slide as a reference.

\subsection{Sample imaging.}

TEM images were acquired with FEI Tecnai 20, operated at 200 kV (FEI Co., Eindhoven, Netherlands). SEM images acquired with FEI Inspect F field emission gun SEM operated at 10-20 kV. Samples were imaged in air with Bruker Dimension Icon Atomic Force Microscope using ScanAssyst-Air probes $(0.4 \mathrm{~N}$ $\mathrm{m}^{-1}$ spring constant) in Peak Force QNM mode. Images were processed by subtracting the background and correcting lines with Gwyddion software. Alignment of wires was characterized using SEM images analyzed with ImageJ software. Statistics for aligned nanowires was collected based on analysis of at least 100 nanowires for each direction.

\section{Acknowledgements}

This work was supported by the University of Georgia internal grant.

\section{References}

1 G. Filipcsei, I. Csetneki, A. Szilágyi and M. Zrínyi, in Oligomers-Polymer Composites - Molecular Imprinting, Springer, Berlin Heidelberg, 2007, pp. 137-189.

2 P. Fratzl, Science, 2012, 335, 177-178. 
3 J. Jestin, F. Cousin, I. Dubois, C. Ménager, R. Schweins, J. Oberdisse and F. Boué, Adv. Mater., 2008, 20, 2533-2540.

4 P. D. Shima and J. Philip, J. Phys. Chem. C, 2011, 115, 20097-20104.

5 J. S. Leng, W. M. Huang, X. Lan, Y. J. Liu and S. Y. Du, Appl. Phys. Lett., 2008, 92, 204101.

6 L. V. Nikitin, G. V. Stepanov, L. S. Mironova and A. I. Gorbunov, J. Magn. Magn. Mater., 2004, 272-276, 20722073.

7 F. Wen-Xiao, H. Zhen-Hui, X. Xue-Qing and S. Hui, Chin. Phys. Lett., 2005, 22, 2386-2389.

8 M. Grzelczak, J. Vermant, E. M. Furst and L. M. LizMarzán, ACS Nano, 2010, 4, 3591-3605.

9 Z. H. Nie, A. Petukhova and E. Kumacheva, Nat. Nanotechnol., 2010, 5, 15-25.

10 R. M. Erb, R. Libanori, N. Rothfuchs and A. R. Studart, Science, 2012, 335, 199-204.

11 Y. Cao, W. Liu, J. Sun, Y. Han, J. Zhang, S. Liu, H. Sun and J. Guo, Nanotechnology, 2006, 17, 2378-2380.

12 M. P. Pileni, J. Phys. Chem. B, 2001, 105, 3358-3371.

13 R. S. M. Rikken, R. J. M. Nolte, J. C. Maan, J. C. M. v. Hest, D. A. Wilson and P. C. M. Christianen, Soft Matter, 2014, 10, 1295-1308.

14 J. Fang, H. Wang, Y. Xue, X. Wang and T. Lin, ACS Appl. Mater. Interfaces, 2010, 2, 1449-1455.

15 M. A. Correa-Duarte, M. Grzelczak, V. Salgueiriño-Maceira, M. Giersig, L. M. Liz-Marzán, M. Farle, K. Sierazdki and R. Diaz, J. Phys. Chem. B, 2005, 109, 19060-19063.

16 K. Kordás, T. Mustonen, G. Tóth, J. Vähäkangas, H. Jantunen, A. Gupta, K. V. Rao, R. Vajtai and P. M. Ajayan, Chem. Mater., 2007, 19, 787-791.

17 M. A. Correa-Duarte, M. Grzelczak, V. Salgueiriño-Maceira, M. Giersig, L. M. Liz-Marzán, M. Farle, K. Sierazdki and R. Diaz, J. Phys. Chem. B, 2005, 109, 19060-19063.

18 S. Kumar, H. Kaur, H. Kaur, I. Kaur, K. Dharamvir and L. M. Bharadwaj, J. Mater. Sci., 2012, 47, 1489-1496.

19 X. Xu, G. Friedman, K. D. Humfeld, S. A. Majetich and S. A. Asher, Chem. Mater., 2002, 14, 1249-1256.

20 A. Tokarev, Y. Gu, A. Zakharchenko, O. Trotsenko, I. Luzinov, K. G. Kornev and S. Minko, Adv. Funct. Mater., 2014, 24, 4738-4745.

21 M. Liu, J. Lagdani, H. Imrane, C. Pettiford, J. Lou, S. Yoon, V. G. Harris, C. Vittoria and N. X. Sun, Appl. Phys. Lett., 2007, 90, 103105.

22 A. K. Bentley, M. Farhoud, A. B. Ellis, A.-M. L. Nickel, G. C. Lisensky and W. C. Crone, J. Chem. Educ., 2005, 82, 765.

23 S.-C. Lin, S.-Y. Chen, Y.-T. Chen and S.-Y. Cheng, J. Alloys Compd., 2008, 449, 232-236.

24 G. De Las Cuevas, J. Faraudo and J. Camacho, J. Phys. Chem. C, 2008, 112, 945-950.

25 K. Mandel and F. Hutter, Nano Today, 2012, 7, 485-487.

26 D. Langley, G. Giusti, C. Mayousse, C. Celle, D. Bellet and J. P. Simonato, Nanotechnology, 2013, 24, 452001.

27 H. Wu, L. Hu, M. W. Rowell, D. Kong, J. J. Cha, J. R. McDonough, J. Zhu, Y. Yang, M. D. McGehee and Y. Cui, Nano Lett., 2010, 10, 4242-4248.
28 L. Hu, H. S. Kim, J.-Y. Lee, P. Peumans and Y. Cui, ACS Nano, 2010, 4, 2955-2963.

29 M.-S. Lee, K. Lee, S.-Y. Kim, H. Lee, J. Park, K.-H. Choi, H.-K. Kim, D.-G. Kim, D.-Y. Lee, S. Nam and J.-U. Park, Nano Lett., 2013, 13, 2814-2821.

30 J.-Y. Lee, S. T. Connor, Y. Cui and P. Peumans, Nano Lett., 2008, 8, 689-692.

31 H. Wu, D. Kong, Z. Ruan, P. C. Hsu, S. Wang, Z. Yu, T. J. Carney, L. Hu, S. Fan and Y. Cui, Nat. Nanotechnol., 2013, 8, 421-425.

32 A. R. Madaria, A. Kumar and C. Zhou, Nanotechnology, 2011, 22, 245201.

33 D. Y. Choi, H. W. Kang, H. J. Sung and S. S. Kim, Nanoscale, 2013, 5, 977-983.

34 S. De, T. M. Higgins, P. E. Lyons, E. M. Doherty, P. N. Nirmalraj, W. J. Blau, J. J. Boland and J. N. Coleman, ACS Nano, 2009, 3, 1767-1774.

35 S. Hong, J. Yeo, G. Kim, D. Kim, H. Lee, J. Kwon, H. Lee, P. Lee and S. H. Ko, ACS Nano, 2013, 7, 5024-5031.

36 S. M. Bergin, Y. H. Chen, A. R. Rathmell, P. Charbonneau, Z. Y. Li and B. J. Wiley, Nanoscale, 2012, 4, 1996-2004.

37 A. Tao, F. Kim, C. Hess, J. Goldberger, R. He, Y. Sun, Y. Xia and P. Yang, Nano Lett., 2003, 3, 1229-1233.

38 A. R. Tao, J. Huang and P. Yang, Acc. Chem. Res., 2008, 41, 1662-1673.

39 A. Tao, F. Kim, C. Hess, J. Goldberger, R. He, Y. Sun, Y. Xia and P. Yang, Nano Lett., 2003, 3, 1229-1233.

40 A. R. Tao, J. Huang and P. Yang, Acc. Chem. Res., 2008, 41, 1662-1673.

41 R. Gunawidjaja, H. Ko, C. Jiang and V. V. Tsukruk, Chem. Mater., 2007, 19, 2007-2015.

42 C. J. Murphy and C. J. Orendorff, Adv. Mater., 2005, 17, 2173-2177.

43 P. A. Smith, C. D. Nordquist, T. N. Jackson, T. S. Mayer, B. R. Martin, J. Mbindyo and T. E. Mallouk, Appl. Phys. Lett., 2000, 77, 1399-1401.

44 Z. Deng and C. Mao, Nano Lett., 2003, 3, 1545-1548.

45 A. J. Mieszawska, G. W. Slawinski and F. P. Zamborini, J. Am. Chem. Soc., 2006, 128, 5622-5623.

46 E. C. Walter, M. P. Zach, F. Favier, B. J. Murray, K. Inazu, J. C. Hemminger and R. M. Penner, ChemPhysChem, 2003, 4, 131-138.

47 C. Xiang, S.-C. Kung, D. K. Taggart, F. Yang, M. A. Thompson, A. G. Güell, Y. Yang and R. M. Penner, ACS Nano, 2008, 2, 1939-1949.

48 M. A. Blesa, H. A. Marinovich, E. C. Baumgartner and A. J. G. Maroto, Inorg. Chem., 1987, 26, 3713-3717.

49 J.-Y. Lee, S. T. Connor, Y. Cui and P. Peumans, Nano Lett., 2008, 8, 689-692.

50 A. R. Madaria, A. Kumar and C. Zhou, Nanotechnology, 2011, 22, 245201.

51 H.-G. Cheong, R. E. Triambulo, G.-H. Lee, I.-S. Yi and J.-W. Park, ACS Appl. Mater. Interfaces, 2014, 6, 78467855.

52 A. R. Madaria, A. Kumar, F. N. Ishikawa and C. Zhou, Nano Res., 2010, 3, 564-573. 
53 A. Carlson, A. M. Bowen, Y. Huang, R. G. Nuzzo and J. A. Rogers, Adv. Mater., 2012, 24, 5284-5318.

54 S. Kirkpatrick, Rev. Mod. Phys., 1973, 45, 574-588.

55 R. M. Mutiso, M. C. Sherrott, J. Li and K. I. Winey, Phys. Lett. B, 2012, 86.
56 C. Grimaldi and I. Balberg, Phys. Rev. Lett., 2006, 96, 066602.

57 A. Celzard and J. F. Marêché, Physica A, 2003, 317, 305-312.

58 W. Wu, Q. He and C. Jiang, Nanoscale Res. Lett., 2008, 3, 397-415. 